\title{
EXISTENCIA DE SOLUCIONES A PROBLEMAS ELÍPTICOS NO LOCALES CON DEPENDENCIA DEL GRADIENTE VÍA LA TÉCNICA DE PASO DE MONTAÑA
}

\author{
Victor E.Carrera Barrantes ${ }^{1}$, Raúl M. Izaguirre Maguiña ${ }^{2}$, Juan B. Bernui Barros ${ }^{3}$ \& Benigno \\ Godoy Torres ${ }^{4}$
}

Resumen: El objetivo principal de este trabajo es estudiar la solubilidad del problema elíptico no local

$$
-M\left(\int_{\Omega}|\nabla u|^{2}\right) \Delta u=f(x, u, \nabla u)
$$

con condición de frontera de Dirichlet cero en un dominio suave y acotado de $\mathbb{R}^{n}$, con $f: \Omega \rightarrow \mathbb{R}$ y $M: \mathbb{R} \rightarrow \mathbb{R}$ como funciones dadas.

Palabras clave: Problemas elípticos no locales, paso de montaña, métodos de iteración.

\section{EXISTENCE OF SOLUTIONS TO NONLOCAL ELIPTIC PROBLEM WITH DEPENDENCE ON THE GRADIENT VIA MOUNTAIN-PASS TECHNIQUES}

Abstract: The main goal of this work is to study the solvability of the nonlocal elliptic problem

$$
-M\left(\int_{\Omega}|\nabla u|^{2}\right) \Delta u=f(x, u, \nabla u)
$$

with zero Dirichlet boundary conditions on a bounded and smooth domain of $\mathbb{R}^{n}$, with $f: \Omega \rightarrow \mathbb{R}$ and $M: \mathbb{R} \rightarrow \mathbb{R}$ are given functions.

Key words: Nonlocal elliptic problems, mountain pass, iteration methods.

\section{Introduction}

The purpose of this article is to investigate the existence of solutions for the nonlocal elliptic problem

$$
\begin{gathered}
-M\left(\int_{\Omega}|\nabla u|^{2}\right) \Delta u=f(x, u, \nabla u) \quad \text { in } \Omega, \\
u=0 \quad \text { on } \partial \Omega,
\end{gathered}
$$

where $\Omega \subset \mathbb{R}^{N}, N \geq 3$ is a bounded smooth domain, $f: \bar{\Omega} \times \mathbb{R} \times \mathbb{R}^{N} \rightarrow \mathbb{R}$ and $M: \mathbb{R} \rightarrow \mathbb{R}$ are given functions. The equation (1.1) is not variational and when $M(t)=1$ was studied by several authors (See :???) using topological degree, methods of sub and supersolutions, etc. So the well developed critical point theory is of no avail for a direct attack to problem (1.1). In the present

\footnotetext{
${ }^{1}$ UNMSM, Facultad de Ciencias Matemáticas. Lima, Perú, c-mail: vacrrerab ayahoo.com

${ }^{2}$ UNMSM, Facultad de Ciencias Matemáticas, Lima, Perú, e-mail: raul_izaguirre2222@iyahoo.es

${ }^{3}$ UNAC, Facultad de Ciencias Matemáticas, Lima, Perú. e-mail: jbermuib @iyahoo.com

${ }^{4}$ UNMSM, Facultad de Ciencias Matemáticas, Lima, Perú, e-mail: bgodoy@ummsm.edu.pe
} 
work we adapt the technique explored by De Figueiredo et al. [5]: we associate with the problem (1.1) a fanily of semilinear elliptic problems with no dependence on the gradient of the solution; this new problems are variational and we can apply the mountain-pass techniques, then we use an iterative scheme.

As it s well known, problem (1.1) is the stationary counterpart of the hyperbolic Kirchhoff equation

$$
\begin{gathered}
\rho u_{t t}-\left[\frac{P_{0}}{+}+\frac{E}{2 L} \int_{0}^{L} u_{x}^{2} d x\right] u_{x x}=0 \quad \text { in }(0, L) \times(0, \infty), \\
u(0, t)=0=u(L, t) \quad \text { on }(0, T), \\
u(x, 0)=u_{0}(x), \quad u_{t}(x, 0)=u_{1}(x) \quad \text { in }(0, L) .
\end{gathered}
$$

that appeared at the first time in the work ok Kirchhoff [??], in 1883. The equation in (1.2) is called Kirchhoff equation and it extends the classical D'alembert wave equation, by considering the effects of the changes in the length of the strings during the vibrations.

The interest of the mathematicians on the so-called nonlocal problems like (1.1), (1.2) (nonlocal because of the presence of the term $M\left(\int_{\Omega}|\nabla u|^{2} d x\right)$ ) has increased because they represent a variety of relevant physical situations and requires a nontrivial apparatus to solve them.

The paper is organized as follows: In section 2, we will give the existence of solutions for the system

$$
\begin{gathered}
-M\left(\int_{\Omega}|\nabla w|^{2}\right) \Delta u=f(x, u, \nabla w) \quad \text { in } \Omega, \\
u=0 \quad \text { on } \partial \Omega,
\end{gathered}
$$

for each $w \in H_{0}^{1}(\Omega)$. In section 3 we will study the solution for (1.1) using a iterative scheme and results of section 2 .

\section{Notations and Preliminaries}

We will denote by $\mathrm{C}$ the general positive constant (the exact value may change from line to line). For convenience, we give the following hypotheses

(H.1) (i) A typical assumption for $M \in C^{1}(0,+\infty)$ is that there exists $m_{0}>0$ such that $M(t) \geq m_{0}$ for all $t \in[0,+\infty[$

(ii) There exists $m_{1}>m_{0}$ such that $M(t)=m_{1} \forall t \geq t_{0}$ for some $t_{0}>0$

(H.2) We suppose that $f: \bar{\Omega} \times \mathbb{R} \times \mathbb{R}^{N} \rightarrow \mathbb{R}$ is a locally Lipschtiz continuous

(i) $\lim _{t \rightarrow 0} \frac{f(x, t, \xi)}{t}=0$ uniformly for all $x \in \bar{\Omega}, \xi \in \mathbb{R}^{N}$

(ii) There exist constants $a_{1}>0$ and $p \in\left(1, \frac{N+2}{N-2}\right)$ such that

$$
|f(x, t, \xi)| \leq a_{1}\left(1+|t|^{p}\right) \quad \forall \xi \in \mathbb{R}^{N}, \quad t \in \mathbb{R} . \quad \xi \in \mathbb{R}^{N}
$$

(iii) There exists constant $\theta>\operatorname{máx}\left\{2, \frac{2}{m_{0}}\right\}$ and $T>0$ such that

$$
0<\theta \quad(x . t . \xi) \leq t f(x, t . \xi) \quad \forall \xi \in \mathbb{R}^{N}, \quad|t| \geq T \in \mathbb{R} . \quad \xi \in \mathbb{R}^{N}
$$

where

$$
(x, t . \xi)=\int_{0}^{t} f(x . s . \xi) d s
$$


(iv) There exist constant $a_{2}, a_{3}>0$ such that

$$
(x, t, \xi) \geq a_{2}|t|^{\theta}-a_{3} \quad \text { for all } x \in \bar{\Omega}, \quad t \in \mathbb{R}, \quad \xi \in \mathbb{R}^{N}
$$

Observation 2.1. From (i) and (ii) it follows that $\theta \leq p+1$

(H.3) The function $\mathrm{f}$ satisfies

$$
\begin{array}{cll}
\text { (i) }\left|f\left(x, t^{\prime}, \xi\right)-f\left(x, t^{\prime \prime}, \xi\right)\right| \leq L_{1}\left|t^{\prime}-t^{\prime \prime}\right| & \forall x \in \bar{\Omega}, t^{\prime}, t^{\prime \prime} \in\left[0, \rho_{1}\left[,|\xi| \leq \rho_{2}\right.\right. \\
\text { (ii) }\left|f\left(x, t, \xi^{\prime}\right)-f\left(x, t, \xi^{\prime \prime}\right)\right| \leq L_{2}\left|\xi^{\prime}-\xi^{\prime \prime}\right| & \forall x \in \bar{\Omega}, t \in\left[0, \rho_{1}\left[,\left|\xi^{\prime}\right|,\left|\xi^{\prime \prime}\right| \leq \rho_{2}\right.\right.
\end{array}
$$

where $\rho_{1}$ and $\rho_{2}$ depend explicitly on $p, N, \theta, a_{1}, a_{2}, a_{3}$ given in the previous hypotheses.

We recall that by a solution of (1.1) we mean a weak solution, that is, a function $u \in H_{0}^{1}(\Omega)$ such that

$$
\int_{\Omega} \nabla u \cdot \nabla d x=\int_{\Omega} \frac{f(x, u, \nabla u)}{M\left(\int_{\Omega}|\nabla u|^{2}\right)} \varphi d x, \quad \text { for all } \varphi \in H_{0}^{1}(\Omega) .
$$

Now, we are in position to establish our main result.

Theorem 1. Assume hypotheses (H.1)-(H.2) hold. Then, there exists positive constants $c_{1}, c_{2}$ such that for each $w \in H_{0}^{1}(\Omega)$ then problem (1.3) has one solution $u_{w}$ such that

$$
c_{1} \leq\left\|u_{w}\right\| \leq c_{2}
$$

where $\|u\|=\left(\int_{\Omega}|\nabla u|^{2}\right)^{1 / 2}$. Moreover, under the above hypotheses, problem (1.3) has a positive and negative solution.

Observation 2.2. It is well known, that if we are looking only positive solutions, we need assumptions (H.2) (iii)-(iv) only for positive $t$.

Theorem 2.Assume (H.1)-(H.3). Then problem (1.1) has a positive and negative solution provided

$$
\frac{4 M_{2} c_{2}^{2}+L_{2} \lambda_{1}^{-1 / 2}}{m_{0}-L_{1} \lambda_{1}^{-1}}<1
$$

where $\lambda_{1}$ is the first eigenvalue of $-\Delta$ and $M_{2}=\max \left\{\left|M^{\prime}(r)\right| ; 0 \leq r \leq c_{2}^{2}\right\}$. Moreover the solutions obtained are of the class $C^{2}$.

\section{Proof of theorem 1 .}

The weak solutions of (1.3) are precisely the critical points of the functional

$$
I_{w}(u)=\frac{1}{2}\|u\|^{2}-\int_{\Omega} H(x, u, \nabla w) d x
$$

where $H(x, u, \nabla w)=\frac{F(x, u, \nabla w)}{M\left(\int_{\Omega}|\nabla w|^{2}\right)}$

We will prove, by steps, that $I_{w}$ has the geometry of the mountain pass theorem, and finally that the obtained solutions have the uniform bounds stated in the theorem.

Step1.Let $w \in H_{0}^{1}(\Omega)$. Then there exists positive numbers $\rho, \alpha>0$ which are independent of $w$ such that

$$
I_{w}(u) \geq \alpha \quad \forall u \in H_{0}^{1}(\Omega):\|u\|=\rho
$$


Proof. By (H.2)(i), given any $\epsilon>0$ there exists $\delta>0$ such that

$$
|H(x, t, \quad)|<\frac{\epsilon t^{2}}{2 m_{0}} \quad \forall|t| \leq \delta
$$

and, by (H.2)(ii), there exists $K=K_{\delta}>0$ such that

$$
|H(x, t, \zeta)|<K|t|^{p+1} \quad \forall|t| \geq \delta
$$

So, using Sobolev Embedding Theorem, we get

$$
I_{w}(u) \geq\left(\frac{1}{2}-\frac{\epsilon}{m_{0} \lambda_{1}}\right)\|u\|^{2}-k_{\epsilon}\|u\|^{p+1}
$$

with $k_{\epsilon}$ a constant independent of $w$. Since $p>1$, the thesis easily follows.

Step2. Let $w \in H_{0}^{1}(\Omega)$. Fix ${ }_{0} \in H_{0}^{1}(\Omega)$ with $\left\|{ }_{0}\right\|=1$. Then there is a $T>0$, independent of $\mathrm{w}$, such that

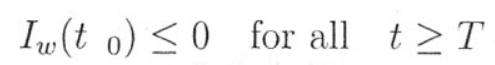

Proof. First, we observe that, from (H.1)(ii) and (H.2)(iii)

$$
|H(x, t, \zeta)| \geq C_{1}|t|^{\theta}-C_{2}, \quad \text { for all } t>t_{0}
$$

So, it follows from (3.4) that

$$
\begin{aligned}
I_{w}\left(t_{0}\right)= & \left.\frac{1}{2} t^{2}\left\|{ }_{0}\right\|^{2}\right)-\int_{\Omega} H\left(x, t_{0}, \nabla w\right) d x \\
& \leq \frac{1}{2} t^{2}-c|t|^{\theta}+C \\
& \rightarrow-\infty \text { as } \quad t \rightarrow+\infty
\end{aligned}
$$

due to $\theta>2$. So, we obtain independent of ${ }_{0}$ and also $w$ that (3.3) holds.

Step3. Let $\left\{u_{n}\right\}$ be a Palais-Smale sequence in $H_{0}^{1}(\Omega)$ that is $I_{w}\left(u_{n}\right) \rightarrow c$ and $I_{w}^{\prime}\left(u_{n}\right) \rightarrow 0$. Then

$$
\begin{aligned}
c+\left\|u_{n}\right\| \geq & I_{w}\left(u_{n}\right)-\frac{1}{\theta}\left\langle I_{w}^{\prime}\left(u_{n}\right) u_{n}\right\rangle \\
& =\left(\frac{1}{2}-\frac{1}{\theta}\right)\left\|u_{n}\right\|^{2}-\underbrace{\int_{\Omega}\left(H\left(x, u_{n}, \nabla w\right)-\frac{1}{\theta} \frac{f\left(x, u_{n}, \nabla w\right)}{M\left(\int_{\Omega}|\nabla w|^{2}\right)} u_{n}\right) d x}_{L}
\end{aligned}
$$

Here, we claim that $\mathrm{L}$ is bounded. Indeed, we consider

$$
\Omega_{n}=\left\{x \in \Omega:\left\|u_{n}(x)\right\|>T\right\}
$$

with $T$ given in (H.2)(iii), then

$$
\begin{aligned}
L= & \underbrace{\int_{\Omega_{n}}\left(H\left(x, u_{n}, \nabla w\right)-\frac{1}{\theta} \frac{f\left(x, u_{n}, \nabla w\right)}{M\left(\int_{\Omega}|\nabla w|^{2}\right)} u_{n}\right) d x}_{L_{1}}+ \\
& \underbrace{\int_{\Omega \backslash \Omega_{n}}\left(H\left(x, u_{n}, \nabla w\right)-\frac{1}{\theta} \frac{f\left(x, u_{n}, \nabla w\right)}{M\left(\int_{\Omega}|\nabla w|^{2}\right)} u_{n}\right) d x}_{L_{2}}
\end{aligned}
$$


But $L_{1} \leq 0$ and

$$
\left|L_{2}\right| \leq \frac{a_{1}|\Omega|}{m_{0}}\left[\left(1+|T|^{p}\right)+\frac{1}{\theta}\left(|T|+\frac{|T|^{p+1}}{p+1}\right)\right]=K
$$

Hence $L \leq K$. So $\left\{u_{n}\right\}$ is bounded in $H_{0}^{1}(\Omega)$, and it admits a weakly convergence subsequence. From the Rellich-Kondrachov Theorem and results of weak convergence, standard argument shows that $\left\{u_{n}\right\}$ admits a strongly convergence subsequence.

Step4. From Steps 1-3 and $I_{w}(0)=0$, I satisfies the conditions of the mountain pass theorem. So $I_{w}$ admits at least one nontrivial critical point $u_{w}$, at an inf max level,which is a weak solution of (1.3), that is

$$
\begin{aligned}
& \text { a) } I_{w}^{\prime}\left(u_{w}\right)=0 \\
& \text { b) } I_{w}\left(u_{w}\right)=\inf _{\gamma \in \Gamma} \operatorname{máx}_{t \in[0,1]} I_{w}(\gamma(t)) \geq \alpha
\end{aligned}
$$

where $\Gamma=\left\{\gamma \in C^{0}\left([0,1], H_{0}^{1}(\Omega)\right): \gamma(0)=0, \gamma(1)=T_{0}\right\}$,for some 0 and $T$ as in Step 2. From now on we fix such a 0 and such a $T$.

Step5. Let $w \in H_{0}^{1}(\Omega)$. There exists a positive constant $c_{1}$, independent of $w$ such that

$$
\left\|u_{w}\right\| \geq c_{1}
$$

for all solution $u_{w}$ obtained in Step 4.

Proof. From the equation (1.3) one gets

$$
\int_{\Omega}\left|\nabla u_{w}\right|^{2} d x=\int_{\Omega} \frac{f\left(x, u_{w}, \nabla w\right)}{M\left(\int_{\Omega}|\nabla w|^{2}\right)} u_{w} d x
$$

By (H.2)(i)-(ii), given $\epsilon>0$, there exists $c_{\epsilon}>0$ independent of $w$, such that

$$
|f(x, t, \nabla w)| \leq \epsilon|t|+c_{\epsilon}|t|^{p}
$$

So, we get

$$
\int_{\Omega}\left|\nabla u_{w}\right|^{2} d x \leq \frac{\epsilon}{m_{0}} \int_{\Omega}\left|u_{w}\right|^{2} d x+c_{\epsilon}^{\prime} \int_{\Omega}\left|u_{w}\right|^{p+1} d x
$$

Hence we have

$$
\left(1-\frac{\epsilon}{\lambda_{1} m_{0}}\right)\left\|u_{w}\right\| \leq \tilde{c_{\epsilon}}\left\|u_{w}\right\|^{p+1}
$$

which implies (3.8) choosing $\epsilon<\lambda_{1} m_{0}$, since $p+1>2$

Step6. There exists a positive constant $c_{2}$ independent of $w$ such that

$$
\left\|u_{w}\right\| \leq c_{2}
$$

Proof. From the infmax characterization of $u_{w}$ in Step4, choosing the path in $\Gamma$ as the segment line joining 0 and 0 , we obtain

$$
I_{w}\left(u_{w}\right) \leq \operatorname{máx}_{t \geq 0} I_{w}\left(\begin{array}{ll}
t & 0
\end{array}\right)
$$

and from (H.3)(iv) we have

$$
\begin{aligned}
\operatorname{máx}_{t \geq 0} I_{w}\left(t_{0}\right) & \leq \operatorname{máx}_{t \geq 0}\left\{\frac{t^{2}}{2}\left\|{ }_{0}\right\|^{2}-a_{2}|t|^{\theta} \int_{\Omega}\left|{ }_{0}\right|^{\theta}+a_{3}|\Omega|\right\} \\
& =c_{2}
\end{aligned}
$$

Therefore we have obtained that

$$
I_{w}\left(u_{w}\right) \leq c_{2}
$$


Here, using the criticality of $u_{w}$ for $I_{w},(3.10)$, (H.2)(iii), one has

$$
\frac{1}{2}\left\|u_{w}\right\|^{2} \leq \hat{c_{2}}+\frac{1}{\theta} \int_{\Omega} \frac{f\left(x, u_{w}, \nabla w\right) u_{w}}{M\left(\int_{\Omega}|\nabla w|^{2}\right)}
$$

Therefore

$$
\left(\frac{1}{2}-\frac{1}{m_{0} \theta}\right)\left\|u_{w}\right\|^{2} \leq \text { const }
$$

The positivity of $u_{w}$ it derives from standard arguments. That is one replaces $f$ by $\hat{f}$ defined as

$$
\hat{f}(x, t, \xi)=\left\{\begin{array}{cc}
f(x, t, \xi) & \text { si } t \geq 0 \\
0 & \text { si } t<0
\end{array}\right.
$$

Here, we observe that $\hat{f}$ still verifies (H.2)(iii)-(iv)( also we take $v_{0}>0$ in step2). So we find a critical point of mountain-pass type for the corresponding functional $\hat{I_{w}}$ that is solution of the problem

$$
\begin{gathered}
-M\left(\int_{\Omega}|\nabla w|^{2}\right) \Delta u_{w}=\hat{f}\left(x, u_{w}, \nabla w\right) \text { in } \Omega, \\
u_{w}=0 \quad \text { on } \partial \Omega,
\end{gathered}
$$

Multiplying the equation by $u_{w}^{-}$and integrating by parts, we conclude that $u_{w}^{-}=0$. Hence $u_{w}^{-}$is positive.

Observation 3.1. In Step 4 we have obtained a weak solution $u_{w}$ of (1.3) for each given $w \in H_{0}^{1}(\Omega)$. Since $p<\frac{N+2}{N-2}$ a standard bootstrap argument, using regularity theory, shows that $u_{w} \in C^{0, \alpha}$ for some $\alpha \in(0,1)$. Now, if $w$ have the additional regularity $w \in C^{1}(\bar{\Omega})$, using the Schauder regularity theory, we show that $u_{w} \in C^{2, \alpha}(\bar{\Omega})$. As a consequence of the Sobolev embedding theorems and Step6 we conclude that, there exist positive constants $\rho_{1}, \rho_{2}$ such that the solution $u_{w}$ satisfies

$$
\left\|u_{w}\right\|_{C^{0}} \leq \rho_{1}, \quad\left\|\nabla u_{w}\right\|_{C^{0}} \leq \rho_{2}
$$

\section{Proof of theorem 2.}

By applying in an iterative way Theorem 1, we construct a sequence $\left\{u_{n}\right\} \subset H_{0}^{1}(\Omega)$ where $u_{n}$ is a solution of the problem

$$
\begin{gathered}
-M\left(\int_{\Omega}\left|\nabla u_{n-1}\right|^{2}\right) \Delta u_{n}=f\left(x, u_{n}, \nabla u_{n-1}\right) \quad \text { in } \Omega, \\
u_{n}=0 \quad \text { on } \partial \Omega,
\end{gathered}
$$

obtained by the mountain pass theorem in theorem 1 . We start from as arbitrary $u_{0} \in$ $H_{0}^{1}(\Omega) \cap C^{1}(\bar{\Omega})$.

By Remark 3.1, we see that

$$
\left\|u_{n}\right\|_{C^{0}} \leq \rho_{1}, \quad\left\|\nabla u_{n}\right\|_{C^{0}} \leq \rho_{2}
$$

Now using (4.1) for $u_{n}$ we get

$$
\begin{aligned}
M\left(\left\|u_{n}\right\|^{2}\right)\left\|u_{n+1}-u_{n}\right\|^{2}= & {\left[M\left(\left\|u_{n-1}\right\|^{2}\right)-M\left(\left\|u_{n}\right\|^{2}\right)\right] \int_{\Omega} \nabla u_{n} \cdot\left(\nabla u_{n+1}-\nabla u_{n}\right) } \\
& +\int_{\Omega}\left[f\left(x, u_{n+1}, \nabla u_{n}\right)-f\left(x, u_{n}, \nabla u_{n}\right)\right]\left(u_{n+1}-u_{n}\right) \\
& +\int_{\Omega}\left[f\left(x, u_{n}, \nabla u_{n}\right)-f\left(x, u_{n}, \nabla u_{n-1}\right)\right]\left(u_{n+1}-u_{n}\right)
\end{aligned}
$$


hence, from (H.1), (H.3). Cauchy-Schwarz and Poincaré inequalities we have

$$
\begin{aligned}
m_{0}\left\|u_{n+1}-u_{n}\right\|^{2} \leq & 4 M_{2} c_{2}^{2}\left\|u_{n}-u_{n-1}\right\|\left\|u_{n+1}-u_{n}\right\| \\
& +L_{1} \lambda_{1}^{-1}\left\|u_{n+1}-u_{n}\right\|^{2}+L_{2} \lambda_{1}^{-1 / 2}\left\|u_{n+1}-u_{n}\right\|\left\|u_{n}-u_{n-1}\right\|
\end{aligned}
$$

Therefore, we conclude that

$$
\begin{aligned}
\left\|u_{n+1}-u_{n}\right\| \leq & \frac{4 M_{2} c_{2}^{2}+L_{2} \lambda_{1}^{-1 / 2}}{m_{0}-L_{1} \lambda_{1}^{-1}}\left\|u_{n}-u_{n-1}\right\| \\
& =: k\left\|u_{n}-u_{n-1}\right\|
\end{aligned}
$$

Since the coefficient $k<1$, we have that $\left\{u_{n}\right\}$ is a Cauchy sequence in $H_{0}^{1}$, and so, $\left\{u_{n}\right\}$ strongly converges in $H_{0}^{1}$ to some function $u \in H_{0}^{1}$.

Since $\left\|u_{n}\right\| \geq c_{1}$, it follows that $u \neq 0$. Hence we find that $u$ is a nontrivial solution of (1.1). By the same argument as in Step6 we have that $u>0$ in $\Omega$. 


\section{REFERENCES}

[1] C. O. Alves \& F. J. S. A. Corrêa, On existence of solutions for a class of problem involving a nonlinear operator, Communications on applied nonlinear analysis, 8(2001). N. 2, 43-56.

[2] C. O. Alves \& D. G. de Figneiredo, Nonvariational elliptic systems via Galerkin methods, Function Spaces, Differential Operators and Nonlinear Analysis - The Hans Triebel Anniversary Volume, Ed. Birkhauser, Switzerland, 47-57, 2003.

[3] A. Ambrosetti, H. Brezis \& G. Cerami, Combined effects of concave and convex nonlinearities in some elliptic problems, J. Funct. Anal. 122(1994), 519-543.

[4] D.G. de Figueiredo, M. Girardi, M. Matzeu, Semilinear elliptic equations with dependence on the gradient via Mountain Pass techniques, Differ. Integ. Eq., 17 (2004) 119-126.

[5] G.M. Figueiredo, Quasilinear equations with dependence on the gradient via Mountain Pass techniques in ${ }^{N}$, Applied Mathematics and Computation 203 (2008) 14-18.

[6] M. Chipot \& J. F. Rodrigues, On a class of nonlocal nonlinear elliptic problems, Mathematical Modelling and Numerical Analysis, Vol. 26, No. 3, 1992, 447-468.

[7] J. L. Lions, Quelques Méthodes de résolution des problémes aux limites non linéaires, Dunod, Gauthier-Vill

[8] C. O. Alves \& F. J. S. A. Corrêa, On existence of solutions for a class of problem involving a nonlinear operator, Communications on applied nonlinear analysis, 8(2001), N. 2, 43-56.

[9] C. O. Alves \& D. G. de Figueiredo, Nonvariational elliptic systems via Galerkin methods, Function Spaces, Differential Operators and Nonlinear Analysis - The Hans Triebel Anniversary Volume, Ed. Birkhauser, Switzerland, 47-57, 2003.

[10] A. Ambrosetti, H. Brezis \& G. Cerami, Combined effects of concave and convex nonlinearities in some elliptic problems, J. Funct. Anal. 122(1994), 519-543.

[11] M. Chipot \& B. Lovat, Some remarks on nonlocal elliptic and parabolic problems, Nonlinear Analysis, T.M.A., Vol. 30, No. 7,(1997), 4619-4627.

[12] M. Chipot \& J. F. Rodrigues, On a class of nonlocal nonlinear elliptic problems, Mathematical Modelling and Numerical Analysis, Vol. 26, No. 3, 1992, 447-468.

[13] J. G. Eisley, Nonlinear vibrations of beams and rectangular plates, Z. Anger. Math. Phys. $15,(1964) 167-175$.

[14] J. Limaco \& L. A. Medeiros, Kirchhoff-Carrier elastic strings in noncylindrical domains, Portugaliae Mathematica, Vol. 14. N. 04,(1999)464-500.

[15] J. L. Lions, Quelques Méthodes de résolution des problémes aux limites non linéaires, Dunod, Gauthier-Villars, Paris, 1969. 